\title{
Inertia from an Asymmetric Casimir Effect.
}

\author{
M.E. McCulloch *
}

February 22, 2013

\begin{abstract}
The property of inertia has never been fully explained. A model for inertia (MiHsC or quantised inertia) has been suggested that assumes that 1) inertia is due to Unruh radiation and 2) this radiation is subject to a Hubble-scale Casimir effect. This model has no adjustable parameters and predicts the cosmic acceleration, and galaxy rotation without dark matter, suggesting that Unruh radiation indeed causes inertia, but the exact mechanism by which it does this has not been specified. The mechanism suggested here is that when an object accelerates, for example to the right, a dynamical (Rindler) event horizon forms to its left, reducing the Unruh radiation on that side by a Rindler-scale Casimir effect whereas the radiation on the other side is only slightly reduced by a Hubble-scale Casimir effect. This produces an imbalance in the radiation pressure on the object, and a net force that always opposes acceleration, like inertia. A formula for inertia is derived, and an experimental test is suggested.
\end{abstract}

\section{Introduction}

Hawking (1975) showed that the event horizon of a black hole can separate paired virtual particles leading to Hawking radiation. It was also proposed by Fulling (1973), Davies (1975) and Unruh (1976) that a similar effect happens to accelerated objects in that a dynamical Rindler event horizon forms on the side they are accelerating away from. This horizon similarly produces radiation so that an accelerated object will perceive a warm background full of blackbody radiation whereas a non-accelerated observer in the same space will see a cold background with no radiation. The Unruh temperature seen by a body with an acceleration 'a' is given by $T=\hbar a / 2 \pi c k$, where $\hbar$ is the reduced Planck's constant, c is the speed of light and k is Boltzmann's constant. This is now called the Fulling-Davies-Unruh effect, or the Unruh effect for short, and it is unclear whether it has been observed or not. It may be the explanation for the observed Sokolov-Turnov effect (Akhmedov and Singleton, 2007).

*SMSE, Plymouth University, Plymouth, PL4 8AA, UK. mike.mcculloch@plymouth.ac.uk 
The property known as inertia has never been adequately explained, and has been rather a neglected part of physics. One model for inertia that uses the electromagnetic part of the Unruh radiation was suggested by Haisch et al. (1994). They proposed that oscillating partons within an accelerated object feel a magnetic Lorentz force, due to their interaction with the zero-point field, that opposes the acceleration of the object. The force they derived from this model was $F=-\Gamma w_{c}^{2} \hbar a / 2 \pi c^{2}$ where $\Gamma$ is the Abraham-Lorentz damping constant of the parton being oscillated, $w_{c}$ is the Compton scale of the parton below which the oscillations of the zero-point field have no effect on it, $\hbar$ is the reduced Planck's constant, a is the acceleration and c is the speed of light. However, although their derived force looks like inertia, their derivation was complex, required the imposition of a high frequency cutoff to avoid infinite energy, and has been criticised on relativistic, and other, grounds, by, for example, Levin (2009).

McCulloch (2007) proposed a model for inertia that could be called a Modification of inertia resulting from a Hubble-scale Casimir effect (MiHsC) or Quantised Inertia. MiHsC assumes that the inertial mass of an object is caused (somehow) by Unruh radiation resulting from its acceleration with respect to surrounding matter, and that this radiation is subject to a Hubble-scale Casimir effect. This means that only Unruh waves that fit exactly into twice the Hubble diameter are allowed, so that an increasingly greater proportion of the Unruh waves are disallowed as they get longer (as acceleration decreases), leading to a new gradual loss of inertia as acceleration reduces. In $\mathrm{MiHsC}$ the inertial mass becomes

$$
m_{I}=m_{g}\left(1-\frac{\beta \pi^{2} c^{2}}{|a| \Theta}\right) \sim m_{g}\left(1-\frac{2 c^{2}}{|a| \Theta}\right)
$$

where $m_{g}$ is the gravitational mass, $\beta=0.2$ (from Wien's displacement law), c is the speed of light, and $\Theta$ is the Hubble diameter $\left(2.7 \times 10^{26} \mathrm{~m}\right.$, from Freedman et al., 2001). For the derivation of Eq. 1 see McCulloch (2007) and for a justification for the use of the modulus of the acceleration see McCulloch (2008b). MiHsC has no adjustable parameters, and predicts cosmic acceleration and galaxy rotation without dark matter (see McCulloch, 2007, 2010, 2012). It violates the equivalance principle, but not in a way that could have been detected in torsion balance experiments (McCulloch, 2011). It has been suggested by Gine (2012) that there may be a link between MiHsC and holographic entropic gravity models (eg: both use the Unruh temperature), but this possible link is not yet clear.

The agreements between MiHsC and anomalous observations provide support to suggest that inertia is (somehow) proportional to the energy in the Unruh radiation spectrum, but an exact mechanism has not been proposed. In this paper for the first time a specific mechanism is suggested. 


\section{$2 \quad$ Method \& Results}

When an object $(\mathrm{O})$ is accelerated to the right as shown in the schematic (Fig. 1), Unruh radiation appears anisotropically and hits the object from all directions, but a dynamic (Rindler) event horizon forms on the left side, since information from the region of space behind this event horizon can never hope to catch up with the object (see the shaded area in the schematic). Now if we calculate the energy density of the Unruh radiation, in the direction of acceleration, to the right, most of the Unruh waves will be allowed by the Hubble-scale Casimir effect (McCulloch, 2007) since the event horizon is far away at the Hubble distance, but on the opposite side, to the left, fewer waves in the Unruh spectrum will be allowed because the dynamic (Rindler) event horizon is much closer, at a distance of $c^{2} / a$ (where a is the acceleration, see eg: Rindler, 2001) so the momentum impact of the Unruh radiation will be lower from the left (with a Rindler-scale Casimir effect) and greater from the right (with a Hubblescale Casimir effect) and this will push the object back against the applied acceleration. This asymetric Casimir effect models inertia intuitively.

The specific calculation can be done as follows. A single particle $(\mathrm{O})$ is considered for simplicity. It is accelerating to the right as shown in the schematic (Fig. 1). The radiation pressure (force) on any small surface area (A) exposed to anisotropic radiation, like Unruh radiation, is

$$
F=\frac{u A}{3}
$$

where $\mathrm{u}$ is the radiation energy density, and $\mathrm{A}$ is the surface area intercepting this radiation, which is assumed to be a small part of the surface area of the whole particle. Now we need to look at the net difference between the force from the left and the right, so to start with, we can consider a line through the particle at an arbitrary angle $\theta$ (see the long dashed line in Fig. 1) and calculate the net force onto the particle along this line from both directions (see arrows) and take its component along the $\mathrm{x}$-axis

$$
d F_{x}=\frac{u_{l e f t} A \cos \theta}{3}-\frac{u_{\text {right }} A \cos \theta}{3}
$$

The energy in the Unruh radiation coming from the right (the second term on the right hand side) is subject to the usual Hubble-scale Casimir effect of McCulloch (2007), so $\left.u_{\text {right }}^{\prime}=u(1-\lambda / 4 \Theta)\right)$, where $\lambda$ is the peak wavelength of the Unruh spectrum. In contrast, the energy of the radiation coming from the left (the first term in the right hand side) is subject to a Rindler-scale Casimir effect with the event horizon now at a much smaller distance away of $c^{2} / a \cos \theta$ (where $a \cos \theta$ is the component of the acceleration in the direction $\theta$ ) so that $u_{\text {left }}^{\prime}=u\left(1-\lambda / 4\left(c^{2} / a \cos \theta\right)\right)$. The difference, the net force in the $\mathrm{x}$-direction, is 


$$
d F_{x}=\frac{u A \cos \theta}{3}\left(1-\frac{\lambda a \cos \theta}{4 c^{2}}-1+\frac{\lambda}{4 \Theta}\right)
$$

This simplifies to

$$
d F_{x}=\frac{u \lambda A \cos \theta}{3}\left(\frac{1}{4 \Theta}-\frac{a \cos \theta}{4 c^{2}}\right)
$$

We now integrate the contribution from all possible angles. To do this we integrate from $\theta=0$ to $\theta=\pi / 2$, then double this to get the result for the $\mathrm{x}-\mathrm{y}$ plane and then integrate this circularily $180^{\circ}$ or $\pi$ around the $\mathrm{x}$ axis (around the angle $\phi$ ) to calculate the total force

$$
\begin{gathered}
d F_{x}=2 \times \frac{u \lambda A}{3} \int_{0}^{\pi} \int_{0}^{\pi / 2}\left(\frac{\cos \theta}{4 \Theta}-\frac{a \cos ^{2} \theta}{4 c^{2}}\right) d \theta d \phi \\
d F_{x}=\frac{2 u \lambda A}{3} \int_{0}^{\pi}\left[\frac{\sin \theta}{4 \Theta}-\frac{a \theta}{8 c^{2}}-\frac{a \sin 2 \theta}{16 c^{2}}\right]_{0}^{\pi / 2} d \phi
\end{gathered}
$$

The circular integral over $\phi$ is equivalent to a multiplication by $\pi$

$$
d F_{x}=\pi \times \frac{2 u \lambda A}{3}\left[\frac{1}{4 \Theta}-\frac{\pi a}{16 c^{2}}\right]
$$

The first term is the brackets is the MiHsC correction to inertia (see McCulloch, 2007) and is tiny compared to the second term, except for low accelerations. Assuming a large acceleration, ie: a terrestrial one, we can neglect this MiHsC term

$$
d F_{x}=-\frac{\pi^{2} u \lambda A a}{24 c^{2}}
$$

Since $u=E / V=h c / \lambda V$ where $\mathrm{V}$ is volume, then

$$
d F_{x}=-\frac{\pi^{2} h A a}{24 c V}
$$

This is taken to be the force on one spherical particle. It implies that the Rindler event horizon that forms in the reference frame of an accelerated particle reduces the energy density of the Unruh radiation in the direction opposite to the acceleration vector, so that it is unable to balance the momentum transferred by the Unruh radiation from the other direction. This produces a net force that 
is always counter to the acceleration (the minus sign in the above formula), and this is a characteristic of inertia.

The ratio $\mathrm{A} / \mathrm{V}$ could be simplified by choosing a distance scale $(\mathrm{x})$ smaller than the particle so that $A / V=x^{2} / x^{3}=1 / x$. Using the Planck distance $\left(l_{P}=1.616 \times 10^{-35} \mathrm{~m}\right)$ as $\mathrm{x}$, for example, then Eq. 10 becomes

$$
d F_{x}=-\frac{\pi^{2} h a}{24 c l_{P}}
$$

and the inertial mass is $m_{i} \sim \pi^{2} h / 24 c l_{P} \sim 5.5 \times 10^{-8} \mathrm{~kg}$ which is about twice the Planck mass $\left(m_{P}=2.176 \times 10^{-8} \mathrm{~kg}\right)$. This derivation is only valid for single particles. To calculate the inertial mass of a compound object it would be necessary to multiply the above mass by the number of particles present. It is also only valid for velocities much slower than that of light, since otherwise Lorentz contraction would alter the dimensions of the particle.

\section{Discussion}

The formula for inertia derived here (Eq. 11) is considerably simpler, in both derivation and final form, than the formula of Haisch et al. (1994) and the mechanism is different. For Haisch et al. (1994) the inertial process was a magnetic Lorentz force acting on particles oscillating at very high frequencies, and to avoid an infinite energy they had to impose an upper frequency limit. The asymetric Casimir effect proposed here produces an energy difference, so no cutoff is needed.

This explanation for inertia depends upon the existence of Unruh radiation, which has not been directly observed. However, the results in this paper, and previous works (McCulloch, 2007-2012) have shown that if one is willing to accept the existence of Unruh radiation, apply Rindler- and Hubble-scale Casimir effects to it, and allow it to have an impact on objects, then certain observed anomalies can be explained simply, and, as shown here, a model for inertia can be derived. These results provide some indirect evidence for Unruh radiation.

The implied dependence of inertia on event horizons suggests a way to test this idea. It was suggested by McCulloch (2008), that it may be possible to modify inertia by creating an event horizon using the metamaterials that were proposed by Pendry et al. (2006) and Leonhardt (2006). They have demonstrated theoretically that radiation of a given wavelength can be bent around an object (which must be smaller than the wavelength) using a metamaterial, making that object invisible to an observer at that wavelength. It may be possible instead, to set up a metamaterial to reflect radiation in such a way that an artificial event horizon is formed. Then according to the model discussed here, this will damp Unruh radiation on one side of the object which would then be accelerated towards the metamaterial. It may be simpler to use metamaterials to more directly damp the Unruh radiation on one side of the object. 


\section{Conclusions}

A new model for inertia (MiHsC or quantised inertia) has been suggested that assumes that 1) inertia is due to Unruh radiation and 2) this radiation is subject to a Hubble-scale Casimir effect. Here, for the first time, a mechanistic model for MiHsC, and inertia, is suggested.

The model assumes that when an object accelerates in one direction, a dynamical Rindler event horizon forms in the opposite direction, producing a Casimir effect, that reduces the Unruh radiation there. As a result, the Unruh radiation pressure on the object is greater from the direction of acceleration, producing a net force that always opposes acceleration, just like inertia.

This model for inertia suggests that if some way could be found to damp the Unruh waves on one side of an object, or create an artificial event horizon on that side (perhaps using metamaterials), the object could then be accelerated in a new way.

\section{Acknowledgements}

Many thanks to T. O'Hare for useful conversations, to the anonymous reviewers for their comments, and to B. Kim for support and encouragement.

\section{References}

Akhmedov, E.T., and D. Singleton, 2007. On the physical meaning of the Unruh effect. JETP Letters, 86(9), 615-619.

Davies, P.C.W., 1975. Scalar production in Schwartzschild and Rindler metrics. J. Phys. A, 8(4), 609.

Freedman, W.L. et al., 2001. Final results from the Hubble Space Telescope key project to measure the Hubble constant. ApJ, 553, 47-72.

Fulling, S.A., 1973. Nonuniqueness of canonical field quantization in Riemannian space-time. Phys. Rev. D., 7(10), 2850.

Gine, J., 2012. The holographic scenario, the modified inertia and the dynamics of the universe. Mod. Phys. Lett. A. Vol. 27, No. 34, 1250208.

Haisch, B., A. Rueda and H.E. Puthoff, 1994. Inertia as a zero-point field Lorentz force. Phys. Rev. A., 49, 678.

Hawking, S., 1974. Black hole explosions. Nature, 248, 30.

Leonhardt, U., 2006. Optical conformal mapping. Science, 312, 1777-1780.

Levin, Y.S., 2009. Inertia as a zero-point field force: critical analysis of the Haisch, Rueda, Puthoff inertia theory. Phys. Rev. A, 79(1), 012114.

McCulloch, M.E., 2007. The Pioneer anomaly from modified inertia. Mon. Not Roy. Astro. Soc., 376, 338. 
McCulloch, M.E., 2008a. Can the flyby anomalies be explained using a modification of inertia? JBIS, 61, 373-378.

McCulloch, M.E., 2008b. Modelling the flyby anomalies using a modification of inertia. MNRAS-letters, 389(1), L57-60.

McCulloch, M.E., 2010. Minimum accelerations from quantised inertia. EPL, 90, 29001.

McCulloch, M.E., 2011. The Tajmar effect from quantised inertia. EPL, 95, 39002 .

McCulloch, M.E., 2012. Testing quantised inertia on galactic scales. Astrophysics and Space Science, 342, 2, 575-578.

Pendry, J.B., D. Schurig, and D.R. Smith, 2006. Controlling electromagnetic fields. Science, 312, 1780-1782.

Rindler, W., 2001. Relativity, special, general and cosmological. Oxford University Press.

Unruh, W.G., 1976. Notes on black hole evaporation. Phys. Rev. D., 14, 870-892.

\section{Figures}

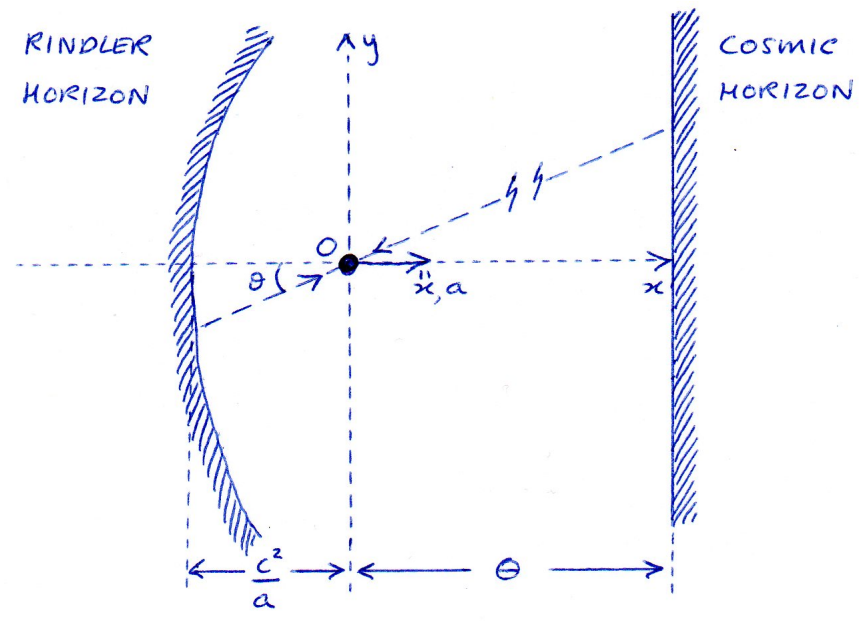

Figure 1. A schematic showing a particle accelerating rightwards $(\mathrm{O})$. The shading shows the cosmic horizon far away to its right (at a distance $\Theta / 2$ ) and a closer Rindler horizon to its left (at a distance $c^{2} / a$ away). This produces an asymetric Casimir effect that pushes the particle $(\mathrm{O})$ to the left against its acceleration: a model for inertia. The lower case $\theta$ shows the angle of integration in the $\mathrm{x}, \mathrm{y}$ plane. 